\title{
Accuracy and Reproducibility of Instrumented Knee-Drawer Tests
}

\author{
Ph. Edixhoven, R. Huiskes, *R. de Graaf, Th. J. G. van Rens, and T. J. Slooff \\ Laboratory of Experimental Orthopaedics, Department of Orthopaedics, and *Department of Mathematics and \\ Statistics, University of Nijmegen, The Netherlands
}

\begin{abstract}
Summary: Instrumented devices for knee-drawer tests have become popular in orthopaedics relatively recently. The objective of the present study was to document the effects of several parameters on the accuracy and reproducibility of anterior-posterior (AP) drawer measurements. An instrumented knee-drawer tester for AP laxity evaluations was constructed, based on the differential displacement method, measuring shifts of the tuberosity relative to the patella. The accuracy of the AP-shift was determined with the parallel use of a highly accurate roentgen stereo photogrammetric (RSP) measurement system on two postmortem leg specimens. The effects of relative motion between patella and femur were negligible. In addition to AP shifts, significant knee flexion and tibial rotations occurred, although the foot and the thigh were fixed as well as possible. The differential displacement method was effective in circumventing this problem. The accuracy of the AP shift was $>10 \%$. The reproducibility of the AP drawer parameters (shifts and compliances) was determined in normal subjects and patients. Tests were made to evaluate the effects of different observers, time sequences, and different days. In addition, effects of muscle relaxation were studied. Overall, the shift parameters at different forces were found to be reproducible to between 5 and $15 \%$. The slopes (compliances) of the laxity curves, at different forces, were found to be reproducible to between 20 and $40 \%$. The reproducibility was principally affected by deviations in the subject positioning procedure. Key Words: Knee instability - Drawer test-Knee ligaments-Knee-joint motion.
\end{abstract}

It is generally accepted that the extent of knee ligament injuries can be estimated by testing kneejoint laxity (10). Although a number of objective measurement methods have been described $(4,6,9,10,12,19)$, diagnosis of knee injury is still based mostly on manual evaluation. A well-known example of these subjective manual tests, exclusively meant to assess the effectiveness of the cruciate ligaments in resisting anterior-posterior (AP)

\footnotetext{
Address correspondence and reprint requests to Dr. R. Huiskes at Laboratory of Experimental Orthopaedics, Department of Orthopaedics, University of Nijmegen, P.O. Box 9101 , $6500 \mathrm{HB}$ Nijmegen, The Netherlands.

Presented in part at the 30th Annual ORS, Las Vegas, 1985 , and at the 4th General Meeting, ESB, Davos, Switzerland, 1984

Dr. Edixhoven's present address is Canisius Hospital, Department of Orthopaedics, Nijmegen, The Netherlands.
}

shift, is the knee-drawer test, executed in $20^{\circ}$ ("Lachman" test), $60^{\circ}$, or $90^{\circ}$ of knee flexion. In all instances, the tibia is pushed backward and pulled forward while the resulting AP displacements of the tibia relative to the femur are estimated, often relative to the contralateral knee joint. An increased anterior drawer indicates an insufficient anterior cruciate ligament (ACL); an increased posterior drawer indicates an insufficient posterior cruciate ligament (PCL).

A number of authors have presented methods for objective evaluations of AP laxity, and a few commercial instruments are available. The methods presented were either based on conventional roentgenographic techniques $(9,10,19)$ or on direct external measurement devices $(4,6,12)$.

Although the principle of the AP laxity measure- 
ment is simple, significant difficulties arise in the execution of the test with instrumented devices. First, because relative displacements between tibia and femur are required, the soft tissue mantles over the bones complicate adequate fixation of external devices to the femur and tibia. Second, although AP drawer is defined as a pure translation, the tibia and the femur can also execute other relative motions, such as axial rotation and flexion, when forced in the AP or PA directions. Clinically, it is not clear whether these additional motions should be suppressed, measured, or simply ignored.

To circumvent the problem of upper leg fixation in the direct measurement methods, the patella is often used as a reference $(4,12,17)$, whereby the AP displacement of the tibial tuberosity is measured relative to the patella. The $\mathrm{KT}-1000$ (Medmetric Corp., San Diego, CA) (4), based on this principle, is fixed to the lower leg and connected to the patella by a displacement sensor. The force is applied manually, and the displacement is read from a dial gauge at specific loads. Markolf and colleagues (12), in their experimental setup, measured the AP displacement of the tuberosity relative to the (fixed) patella-femoral complex with a displacement sensor, while manually pushing or pulling a load-cell attached to the lower leg. The Genucom (Faro, Far Orthopedics, Inc., Montreal, Canada) (6) then measures the full three-dimensional motions of the tibia relative to the femur, using statistical correction methods in the numerical data evaluation to compensate for inadequate fixation.

This article reports the effects of various vari- ables on the accuracy and reproducibility of the AP drawer in normal and cruciate-deficient knees. The information provided is relevant for differential measuring methods in general, as applied in various devices.

\section{MATERIALS AND METHODS}

Test Device

The instrument developed for the present investigation also uses the differential measuring method, whereby the AP displacements of both the tuberosity and the patella are measured relative to an external frame and subtracted (Fig. 1). Each leg is subsequently positioned in $\sim 30^{\circ}$ of hip flexion, $\sim 25^{\circ}$ of knee flexion, and neutral tibial rotation. The upper leg is secured with a thigh holder, and the foot is held in neutral flexion in a foot holder. Displacement transducers are positioned on the tuberosity and on the patella. The displacement feelers are positioned on the patella and the tuberosity by small sliding plates, hence only vertical motions are transferred (see detail, Fig. 1). AP forces are administered manually with a spindle, between $\pm 250 \mathrm{~N}$, and measured with a load-cell. The displacement transducers and the load-cell are connected to a microcomputer, which evaluates the differential displacement (the drawer shift) and the force on-line, and displays these values as a dot in a force-displacement coordinate system on the monitor. Due to restrictions in the computer system, the resolution of the displacement registration is lim-
$1 A, B$

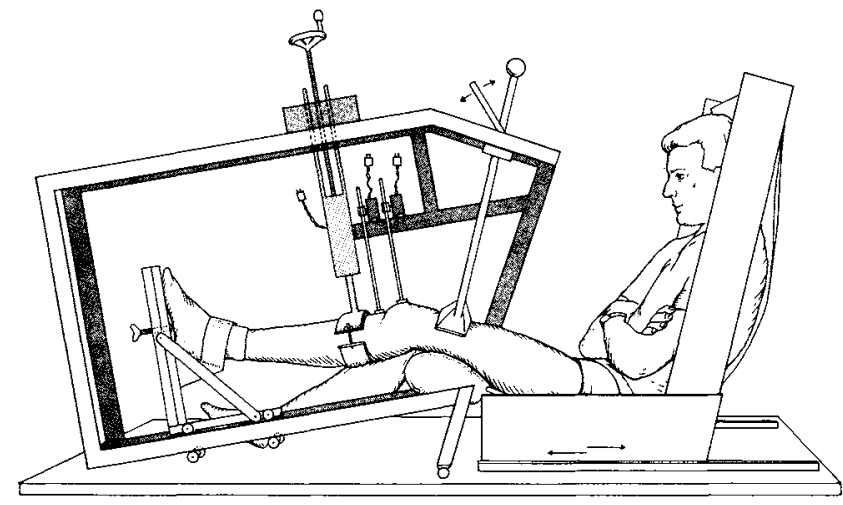

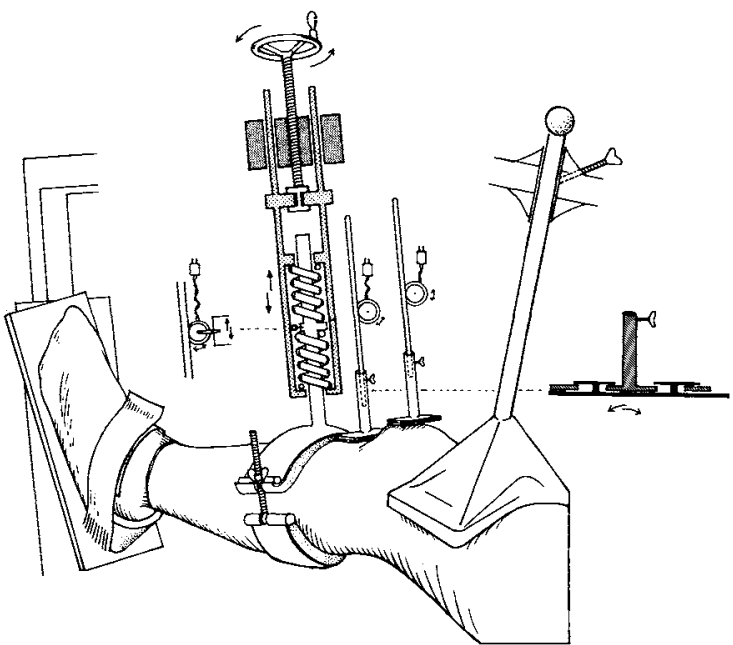

FIG. 1. Schematic drawing of the AP drawer tester (A) and some details (B) of the load application device with (spring-loaded) force measurement, the displacement feelers with low-friction sliding plates, and the thigh holder. 
ited to $0.35 \mathrm{~mm}$, and the resolution of the force registration is limited to $2.2 \mathrm{~N}$. The displacement transducers exert a constant force of $2 \mathrm{~N}$.

Before the actual measuring procedure starts, a complete AP cycle is made (force from zero to 250 $\mathrm{N}$ anterior to $250 \mathrm{~N}$ posterior to zero), both for preconditioning of the joint (discussed later) and to determine whether the patient is at ease. Then, with no force applied on the leg, the dot on the monitor is positioned at the zero-shift position in the coordinate system by adjustment of the displacement transducers. During the actual measuring procedure, two full AP cycles are made, requiring $\sim 50$ s/cycle. This time the force and the shift are registered at 100 discrete points on the monitor and on a floppy disc. The measuring points on the monitor are connected by straight lines to form force-displacement curves (Fig. 2). The data on the floppy disc are later transferred to a mainframe computer for combined data evaluation and statistical analysis.

\section{Data Evaluation}

The applied AP force is denoted by $F_{a}(\mathrm{~N})$-positive in the anterior direction, negative in the poste-

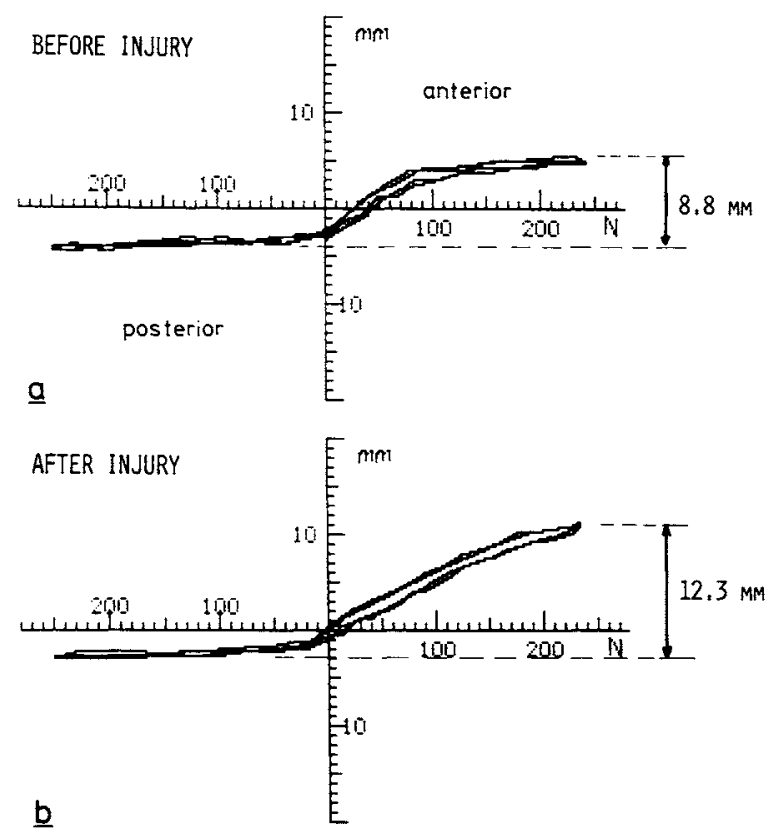

FIG. 2. Load (N)-displacement $(\mathrm{mm})$ curves, as registered on the monitor. (a) Normal knee; (b) same knee after sports injury (anterior cruciate rupture, obtained by coincidence). Lower curves in each graph: posterior-to-anterior-directed shifts. Upper curves: anterior-to-posterior-directed shifts. Although coinciding in most regions, each curve was registered twice. rior direction. The shift is denoted by $s(\mathrm{~mm})$. Because of the weight of the lower leg $(W)$, working in the posterior direction, the real AP force $(F)$ is lower than the applied force: $F=F_{\mathrm{a}}-W . W$ is estimated uniformly as $30 \mathrm{~N}(20)$.

The objective of the data evaluation procedure is to reduce the 100 measuring points (force-shift combinations) describing the force-displacement relation $s=s(F)(-280 \mathrm{~N} \leqslant F \leqslant 220 \mathrm{~N})$ to a reduced number of parameters to facilitate statistical analysis. The shifts $s=s(F)$ were calculated for $F$ $= \pm 90 \mathrm{~N}$ and $F= \pm 180 \mathrm{~N}$. In addition, the compliances $c=c(F)(\mathrm{mm} / \mathrm{N})$ of the knee, i.e., the slopes $c=d s / d F^{\prime}$ were calculated for $F=0$ and $F$ $= \pm 180 \mathrm{~N}$. The compliance is the inverse of the stiffness.

Because two full AP cycles are made (twice the cycle zero to anterior, anterior to posterior, and back to zero), four branches of the force-shift curve are obtained, as shown in Fig. 2. Each cycle produces a lower branch, $s_{1}(F)$, representing the posterior-to-anterior-directed shift, and an upper branch, $s_{u}(F)$, representing the anterior-to-posterior-directed shift. These branches are divided by a hysteresis loop. The slopes of these curves are denoted by $c_{1}(F)$ and $c_{u}(F)$, respectively.

The parameters studied are the lower zero-force shift and the zero-force hysteresis:

$$
s_{o}=s_{1}(0) \text { and } H_{o}=s_{u}(0)-s_{1}(0),
$$

the mean anterior and posterior shifts relative to the lower zero-force shift

$$
S_{F}=1 / 2\left\{s_{u}(F)+s_{1}(F)\right\}-s_{1}(0),
$$

for $F= \pm 90 \mathrm{~N}$ and $F= \pm 180 \mathrm{~N}$, the mean total shifts between $F=90 \mathrm{~N}$ and $F=-90 \mathrm{~N}$ and between $F=180 \mathrm{~N}$ and $F=-180 \mathrm{~N}$

$$
\begin{gathered}
T_{90}=\left|S_{90}\right|+\left|S_{-90}\right|, \\
T_{180}=\left|S_{180}\right|+\left|S_{-180}\right|,
\end{gathered}
$$

the zero-force compliances

$$
C_{u 0}=c_{u}(0) \text { and } C_{10}=c_{1}(0),
$$

and the mean compliances

$$
C_{F}=1 / 2\left\{c_{u}(F)+c_{1}(F)\right\}
$$

for $F= \pm 180 \mathrm{~N}$.

To calculate estimates for these parameters, intervals around the force values $\pm 180 \mathrm{~N}, \pm 90 \mathrm{~N}$, and $0 \mathrm{~N}$ are considered (Fig. 3). The measuring points with respect to these intervals are used to 

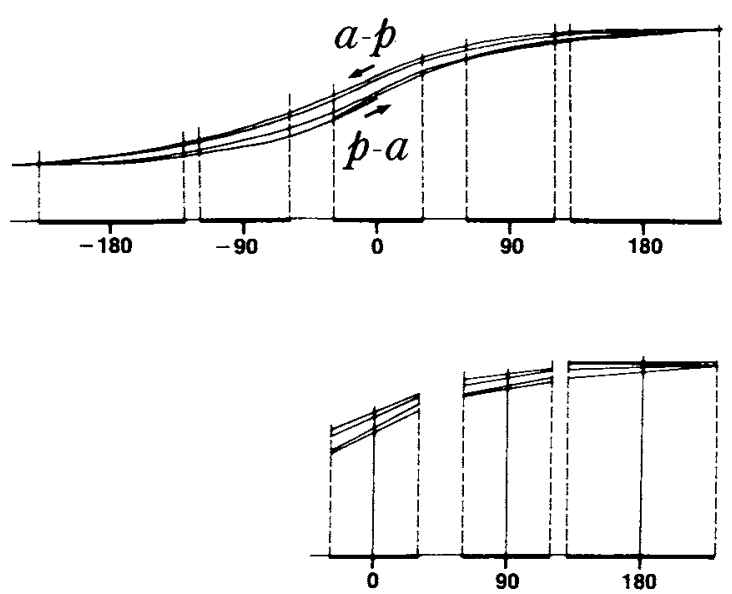

FIG. 3. Partition of load-displacement curve into five intervals around the (gravity-corrected) force values $\pm 180 \mathrm{~N}$, $\pm 90 \mathrm{~N}$, and zero.

estimate the shifts $\left[s_{u}(F), s_{1}(F)\right]$ and slopes $\left[c_{u}(F)\right.$, $\left.c_{1}(F)\right]$ for the force values mentioned and for each of the cycles separately, using a distribution-free approach based on linearization of the corresponding curve segments. For any combination of two sufficiently different measuring points, the slope and the shift values are calculated. The medians of these slope values (18) and shift values are determined as the intended estimates. Before the parameters according to Equations (1) through (7) are calculated, the estimated values $s_{u}(F), s_{1}(F)$, $c_{u}(F)$, and $c_{1}(F)$ are averaged with respect to the two loading cycles, which are thus considered indentical.

\section{Accuracy Test}

Accuracy tests were carried out on two full-leg amputation specimens, using a highly accurate roentgen-stereophotogrammetric (RSP) measurement system $(8,11,16)$ to provide the reference data for the three-dimensional relative motions of the tibia relative to the femur, parallel to the AP laxity tester. Six tantalum markers each $(0.5-$ to $0.8-\mathrm{mm}$ diameter) were inserted in the femur, the tibia, and the patella, using a syringe. The leg was positioned in the laxity tester; that is, the femur was loosely fixed to the table, simulating the feasible motions of the hip joint, and the quadriceps muscle was loaded with $15-$ to $20-\mathrm{N}$ weights. After each AP loading step, the AP shift and the load were registered as measured by the laxity tester. In addition, stereo roentgen exposures were made. These were later measured and evaluated to determine the spatial coordinates of the markers. Using principles of rigid-body kinematics, the relative three-dimensional translations and Euler rotations of the bones were determined with an accuracy of $0.1 \mathrm{~mm}$ and $0.5^{\circ}$, respectively (11). These results were used as the true standard for the accuracy of the laxity tester. Each leg was tested with the intact specimens (experiments $1 \mathrm{~A}$ and $2 \mathrm{~A}$ ), repeated after cutting of the ACL (experiments $1 \mathrm{~B}$ and $2 \mathrm{~B}$ ) and the PCL (experiments $1 \mathrm{C}$ and $2 \mathrm{C}$ ), respectively.

To describe the relative spatial rotations and translations of the bones, three coordinate systems were defined, each with a specific base point (Fig. 4). In the femur, this point $\left(P_{f}\right)$ was chosen in the midsagittal plane, approximately where the helical motion axes are located in flexion of the knee (1). In the tibia, a point $P_{t}$ was chosen on the tuberosity, and in the patella a point $P_{p}$ was chosen in the center of the anterior facet. Relative rotations were expressed in the body-fixed coordinate systems of each bone, subsequently about the $x$-axis (flexion), the $y$-axis (axial rotation) and the $z$-axis (valgusvarus rotation). Because the rotations are small, the sequence is less important in this case. The AP translation of the patella relative to the femur was calculated as the displacement of point $P_{f}$ of the femur relative to a fixed patella in the direction of the $z_{3}$-axis. The proximal-distal translation of the patella as the displacement in the $y_{3}$-axis direction. The AP translation of the tibia relative to the femur, the AP drawer, was calculated as a displacement of the point $P_{f}$ relative to a fixed tibia in the direction of the $z_{2}$-axis.

\section{Reproducibility Tests}

Tests were made to evaluate the reproducibility of the AP drawer parameters as measured with the

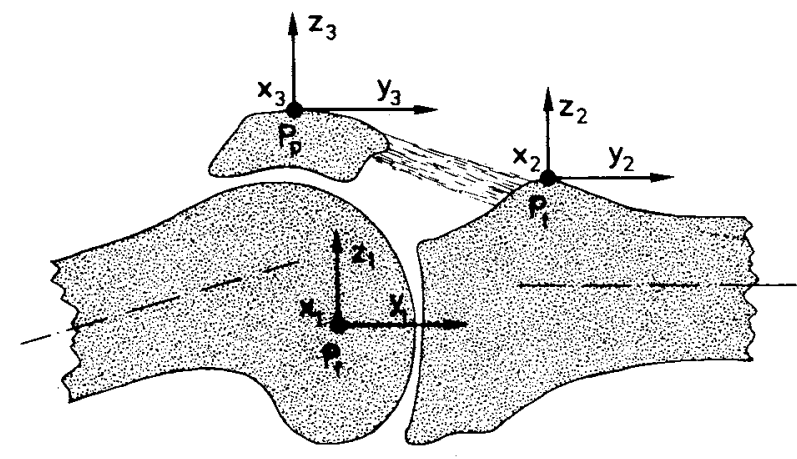

FIG. 4. Bony-fixed reference systems defined in the femur $\left(x_{1}, y_{1}, z_{1}\right)$, the tibia $\left(x_{2}, y_{2}, z_{2}\right)$, and the patella $\left(x_{3}, y_{3}, z_{3}\right)$ to describe the relative motions measured with the roentgen stereo photogrammetric system. 
laxity tester in normal subjects and patients. These tests were done partly by different observers and on different days. In addition, the effects of muscle relaxation were studied.

Tests repeated five times were performed on both knees of 15 individuals (12 normal subjects and 3 patients with unilateral ligament deficiencies) by one observer. Two individuals (four knees) were tested six times a day on 10 or 11 different days, each individual being tested by a different observer. This test was meant to examine the possible existence of additional between-day variation in the parameters. Using one-way analyses of variance (random model), separately for each knee and for each parameter, the hypothesis was tested that the theoretical day means were equal on different days (a theoretical day mean is the mean that would be obtained if an infinite number of tests were done on the day under consideration).

One patient (one stable and one unstable knee) was measured (on one day) five times each by five different observers. As in the previous cases, the test subjects were repositioned in the laxity tester for each new measurement. This experiment was designed to investigate the effects of both the factor of observer and the factor of time of measurement. The observers rotated in a fixed schedule, divided in five periods of the day. First, two-way analyses of variance (fixed model) were applied. Then, by means of one-way analyses of variance, the overall hypothesis of equal theoretical observer means was tested for each parameter. If this hypothesis was rejected $(p \leqslant 0.05)$, it was followed by a multiple comparison analysis according to Scheffé (15) to examine pairwise differences between the observers. Finally, seven patient-volunteers with normal knees were measured before and after receiving lumbar anesthesia, causing complete motor block relaxation Bromage Scale I (2), for other purposes.

\section{RESULTS}

We found repeatedly that preconditioning of the knee to some extent is necessary while applying the laxity tester. When we performed subsequent tests, the second and subsequent cycles of the drawer curve were always reproducible, but the first cycle was always notably different. For that reason, a full precycle, without registration, was always made before the actual test began. Hysteresis between the AP- and the PA-directed parts of the curve (the upper and the lower curves) is always present.

Patient relaxation was important. By quadriceps activation, the subject could produce AP motions, visible on the monitor. Relaxation was checked manually by palpation of the quadriceps during load application. The inclusion of two loading cycles in each test provided an immediate check.

\section{Accuracy Tests}

The AP drawer shifts as determined for specimen 2 (Fig. 5) were compared with the RSP results for the intact knee, then after ACL cutting, and after both ACL and PCL cutting. These curves were not corrected for gravity. As shown in Fig. 5A, the anterior shift in the intact knee was overestimated by $\sim 0.4 \mathrm{~mm}$ or $7 \%$ maximally; the posterior shift was overestimated by $\sim 1 \mathrm{~mm}$ or $20 \%$. In the ACL- and PCL-deficient knees, the anterior error was larger than the posterior one (Fig. 5B and C) and, relative to the value of the drawer, the errors were lower.

Although both the foot and the thigh were fixed as well as possible in the knee-laxity tester, significant amounts of flexion (Fig. 6A) and tibial rotations (Fig. 6B) occurred, the total amounts of which again depended on the presence of the ligaments. When pulled anteriorly, the (in vitro) knee flexed $\sim 6-11^{\circ}$ in this case and rotated internally $11-13^{\circ}$. When the knee was pushed posteriorly, these amounts varied between $\sim 3$ and $4^{\circ}$, and 2 and $6^{\circ}$. These curves were not corrected for gravity.

AP motion of the patella relative to the femur may have contributed to the measurement error. In the RSP measurement, however, this error was extremely small.

The relative motions in specimen 1 followed approximately the same patterns. Total translations and rotations for both specimens between (gravitycorrected) AP forces of $F= \pm 180 \mathrm{~N}$ are summarized in Table 1. In both cases, the laxity tester (column 1) gave a reasonably accurate approximation of the RSP value for drawer shift (column 2). Errors were $\sim 10 \%$ and did not appear to be greatly influenced by knee flexion and tibial rotation (columns 3 and 4). Some AP displacements of the patella relative to the femur occurred (column 5): $\sim 1-3 \%$ of the measured AP shift. The proximaldistal displacements of the patella relative to the femur (column 6) were much larger, due to knee flexion, but because of skin/bone shifts and low friction sliding of the displacement sensor over the 


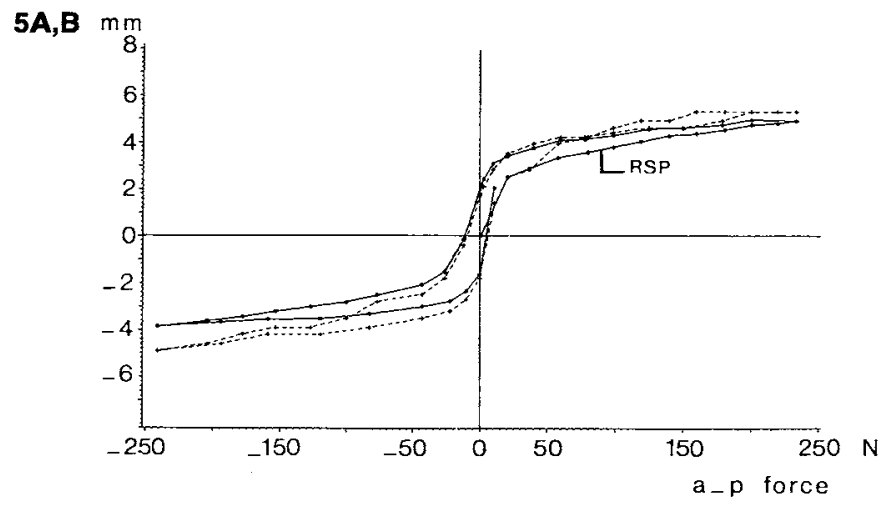

$5 \mathrm{C}$

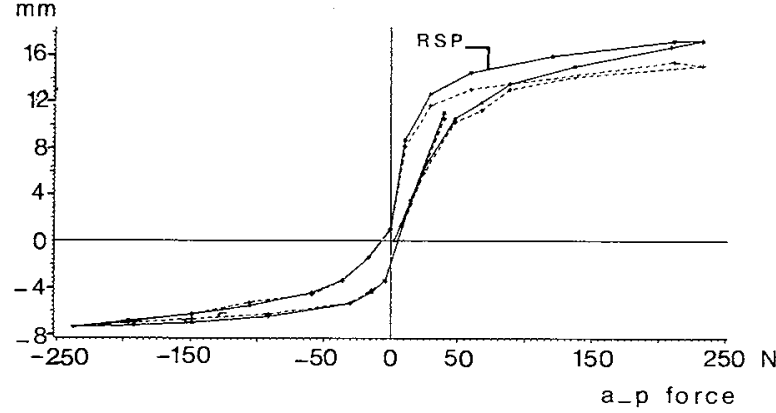

patella hardly affected the measurements of the AP shift. Evidently, the fixation of the upper leg to the frame was more flexible in specimen 2 as compared with specimen 1 (columns 7, 8, and 9), which explains the increased values for knee flexion (column 3).

\section{Reproducibility Tests}

Table 2 shows the results for different parameters from 12 normal subjects, each measured five times

6A,B dgrs

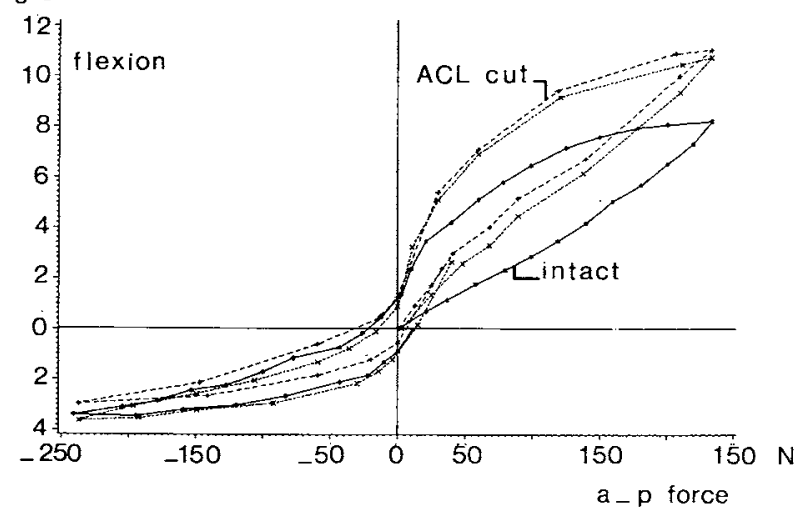

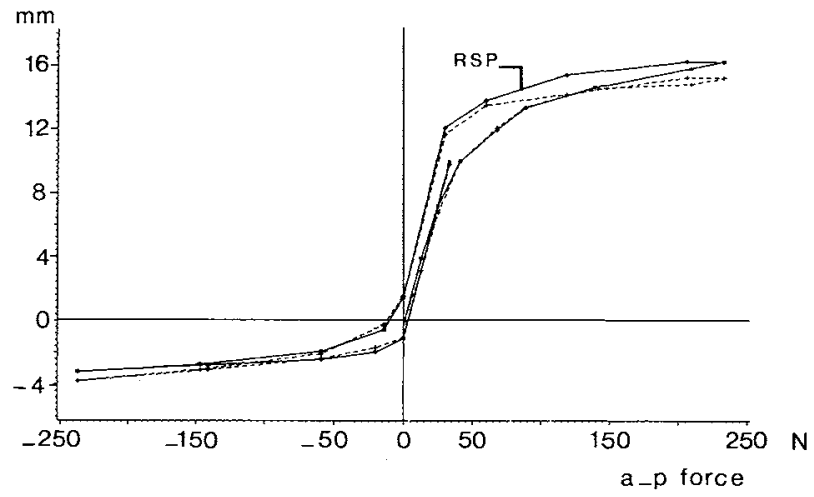

FIG. 5. Comparison between AP drawer shifts as measured with the laxity tester and as found in the roentgen stereo photogrammetric (RSP) evaluation in specimen 2. (A) intact knee; (B) after cutting anterior cruciate ligament (ACL); (C) after cutting $A C L+$ posterior cruciate ligament (PCL). The curves are not corrected for gravity. $=$ roentgen stereo photogram (central knee); $\dagger=$ stress apparatus.

on the same day by one observer. The means and $\mathrm{SD}$ and the absolute coefficients of variation (CV $=\mid \mathrm{SD} /$ mean $\mid \times 100 \%$ ) of one (typical) normal knee are given. Also shown are the means and SD with respect to the average $\mathrm{CV}$ values of both knees of all normals. The total shifts $T_{90}$ and $T_{180}$ displayed the highest reproducibility, with a $\mathrm{CV}$ between 2 and $8 \%$. Second best were the anterior shifts $S_{90}$ and $S_{180}$ with a CV between 3 and $18 \%$, followed by the posterior shifts $S_{-90}$ and $S_{-180}$ between $5 \%$ and 23\%. Much less reproducible were the compliances

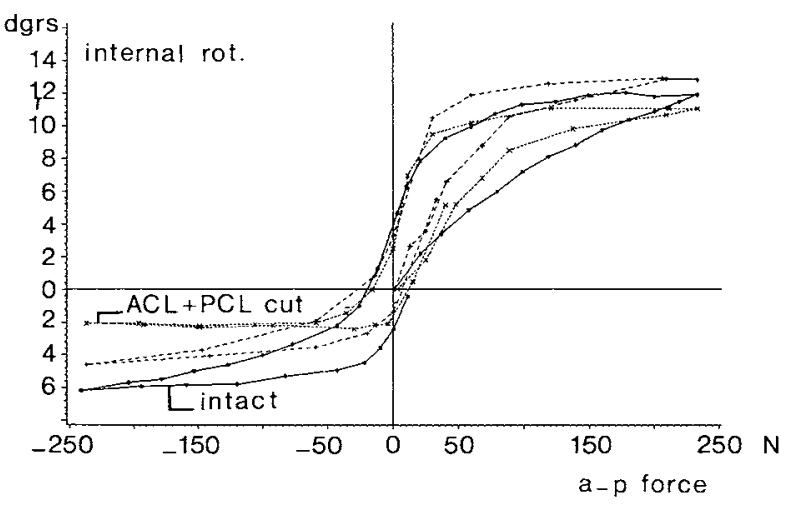

FIG. 6. Flexion-extension (A) and tibial rotation (B) as evaluated with the roentgen stereo photogrammetric (RSP) system in specimen 2, during the drawer test, for the intact specimen and after subsequent cutting of the ligaments. ${ }^{*}=$ intact; $+=A C L$ cut; $X=A C L+P C L$ cut. 
TABLE 1. Comparison between AP drawer shifts measured with laxity tester and the high-accuracy RSP system in the two knee specimens

\begin{tabular}{|c|c|c|c|c|c|c|c|c|c|}
\hline & \multirow{3}{*}{$\frac{\text { Laxity tester }}{\begin{array}{c}\text { AP drawer } \\
(\mathrm{mm})\end{array}}$} & \multirow{2}{*}{\multicolumn{3}{|c|}{ RSP: Tibia (femur) }} & \multirow{2}{*}{\multicolumn{2}{|c|}{$\begin{array}{l}\text { RSP: Patella motion } \\
\text { (femur) }\end{array}$}} & \multirow{2}{*}{\multicolumn{3}{|c|}{ RSP: Absolute motion femur }} \\
\hline & & & & & & & & & \\
\hline & & $\begin{array}{l}\text { AP-transl. } \\
\quad(\mathrm{mm})\end{array}$ & $\begin{array}{l}\text { Flexion } \\
\left({ }^{\circ}\right)\end{array}$ & $\begin{array}{l}\text { Tib.rot. } \\
\left({ }^{\circ}\right)\end{array}$ & $\begin{array}{l}\text { AP-transl. } \\
(\mathrm{mm})\end{array}$ & $\begin{array}{l}\text { transl. } \\
(\mathrm{mm})\end{array}$ & $\begin{array}{l}\text { AP-transl. } \\
\quad(\mathrm{mm})\end{array}$ & $\begin{array}{l}\text { Flex.rot. } \\
\left({ }^{\circ}\right)\end{array}$ & $\begin{array}{l}\text { Axial rot. } \\
\left({ }^{\circ}\right)\end{array}$ \\
\hline & 1 & 2 & 3 & 4 & 5 & 6 & 7 & 8 & 9 \\
\hline \multicolumn{10}{|l|}{ Intact (A) } \\
\hline Spec. 1 & 10.5 & 9.3 & 3.9 & 6.4 & - & - & 8.3 & 2.0 & 0.2 \\
\hline Spec. 2 & 9.0 & 8.2 & 10.6 & 16.6 & 0.3 & 2.6 & 27.0 & 5.6 & 3.5 \\
\hline \multicolumn{10}{|c|}{$A C \tilde{L}$ cut $(B)$} \\
\hline Spec. 1 & 21.2 & 19.2 & 5.7 & 7.6 & 0.2 & 1.6 & 10.1 & 2.6 & 1.9 \\
\hline Spec. 2 & 18.3 & 19.0 & 12.6 & 16.9 & 0.2 & 1.4 & 28.2 & 5.8 & 6.9 \\
\hline \multicolumn{10}{|c|}{$\mathrm{ACL}+\mathrm{PCL}(\mathrm{C})$} \\
\hline Spec. 1 & 23.8 & 23.6 & 5.8 & 9.7 & 0.7 & 1.2 & 7.7 & 2.3 & 1.4 \\
\hline Spec. 2 & 22.8 & 23.3 & 12.0 & 13.3 & 0.5 & 1.4 & 25.9 & 5.5 & 4.1 \\
\hline
\end{tabular}

AP, anterior-posterior; RSP, roentgen stereo photogrammetric; transl., translation; Tib. rot., tibial rotation; Pr-dist. transl., proximal-distal translation; Flex. rot., flexion rotation; Axial rot., axial rotation; ACL, anterior cruciate ligament; PCL, posterior cruciate ligament; Spec., specimen.

Column $1, \mathrm{~T}_{180}$.

at $\pm 180 \mathrm{~N}$, with mean relative variations of $41 \%$ (posterior) and $28 \%$ (anterior). $H_{0}$ was too small for a meaningful determination of the CV. $H_{0}$ was reasonably reproducible per knee, varying in SD from $\sim 0.1$ to $0.3 \mathrm{~mm}$. The zero-force shift $\left(S_{0}\right)$ had a maximal SD value for each knee of $0.9 \mathrm{~mm}$.

For the unstable knees $(n=3)$, tested in the same way, comparable results were obtained. The $\mathrm{SD}$ values were of equal magnitude as compared

TABLE 2. Results of reproducibility tests of 12 normal subjects (24 knees)

\begin{tabular}{|c|c|c|c|c|c|}
\hline \multirow[b]{2}{*}{ Parameter } & \multicolumn{3}{|c|}{$\begin{array}{l}\text { One typical normal knee } \\
\quad(\text { A.B.R. } / \mathbf{n}=5)\end{array}$} & \multicolumn{2}{|c|}{$\begin{array}{l}12 \text { Normal sub- } \\
\text { jects } \\
\text { (average CV of } \\
\text { both knees) }\end{array}$} \\
\hline & $\begin{array}{l}\text { Mean } \\
(\mathrm{mm})\end{array}$ & $\begin{array}{c}\mathrm{SD} \\
(\mathrm{mm})\end{array}$ & $\begin{array}{l}\mathrm{CV} \\
(\%)\end{array}$ & $\begin{array}{l}\text { Mean } \\
(\%)\end{array}$ & $\begin{array}{l}\mathrm{SD} \\
(\%)\end{array}$ \\
\hline \multicolumn{6}{|l|}{ Shift } \\
\hline $\mathrm{T}_{90}$ & 5.6 & 0.2 & 3 & 5 & 1 \\
\hline $\mathrm{T}_{180}$ & 7.0 & 0.3 & 4 & 5 & 2 \\
\hline$S_{-180}$ & -3.0 & 0.4 & 13 & 12 & 4 \\
\hline$S_{-90}^{-180}$ & -2.3 & 0.3 & 12 & 13 & 5 \\
\hline$S_{0}^{-90}$ & - & 0.8 & - & - & - \\
\hline$H_{0}$ & 1.1 & 0.1 & - & - & 一 \\
\hline$S_{90}$ & 3.2 & 0.2 & 6 & 9 & 5 \\
\hline$S_{180}$ & 4.0 & 0.2 & 4 & 7 & 3 \\
\hline Compliance & $\left(10^{-4} \mathrm{~mm} / \mathrm{N}\right)$ & $\left(10^{-4} \mathrm{~mm} / \mathrm{N}\right)$ & & & \\
\hline$C_{-180}$ & 66 & 19 & 29 & 41 & 16 \\
\hline$C_{t \omega 0}$ & 512 & 69 & 13 & 16 & 6 \\
\hline$C_{10}$ & 381 & 43 & 11 & 17 & 6 \\
\hline$C_{\mathrm{L} 80}$ & 68 & 20 & 30 & 28 & 10 \\
\hline
\end{tabular}

Each subject was measured five times on the same day by one observer. with those of normal knees. The absolute CVs tended to be considerably lower, because the shifts and compliances were higher for these knees.

Table 3 shows the results of the measurements with the right knees of the two individuals tested six times a day on 10 or 11 different days. Shown are the overall mean values of the parameters, pooled estimates for the within-day variations (corresponding to the SD values of Table 2), estimates for the between-day variations, and the $\mathrm{p}$ values obtained by testing for each parameter the hypothesis that theoretical day means were equal on different days. Evidently, this hypothesis must be rejected for most of the parameters of knee $1(p \leqslant$ 0.05 ), but not for those of knee 2 . The other two knees followed the same patterns as knee 1 . In particular, with regard to compliance parameters, the between-day variations were usually less important than the within-day variations.

The results of the measurements repeated five times on one day by five different observers on one patient with a normal right knee and a cruciate-deficient left knee are given in Table 4, with respect to five of the parameters. Other parameters showed similar results. Two-way analyses of variance did not reveal clear differences between five time periods during the day. Consequently, a possible time effect was discarded. There are obviously observer effects both with respect to shift parameters and compliances (Table 4). Significant $(p \leqslant 0.05)$ or nearly significant $(0.05<\mathrm{p} \leqslant 0.10)$ differences in pairs were found for several parameters, in most of 
TABLE 3. Means, within-day variances, and estimates for between-day variances in two (right) knees of normal subjects

\begin{tabular}{lcccc}
\hline & \multicolumn{3}{c}{ Normal knee $1(\mathrm{n}=66) /$ normal knee $2(\mathrm{n}=60)$} \\
\cline { 2 - 5 } Parameter & Mean & $\begin{array}{c}\text { W-D } \\
\text { variance } \\
\text { (SD) }\end{array}$ & $\begin{array}{c}\text { B-D } \\
\text { variance }\end{array}$ & p value \\
\hline Shift (mm) & & & & \\
$\mathrm{T}_{90}$ & $4.5 / 4.8$ & $0.3 / 0.4$ & $0.2 / 0.1$ & $0.001 / 0.30$ \\
$\mathrm{~T}_{180}$ & $5.9 / 5.8$ & $0.3 / 0.4$ & $0.3 / 0.2$ & $0.001 / 0.07$ \\
$S_{-180}$ & $-2.7 /-1.8$ & $0.2 / 0.4$ & $0.1 / 0.1$ & $0.001 / 0.30$ \\
$S_{-90}$ & $-2.0 /-1.5$ & $0.2 / 0.4$ & $0.1 / 0.0$ & $0.001 / 0.41$ \\
$S_{0}$ & $\overline{5}$ & $0.4 / 0.8$ & - & - \\
$H_{0}$ & $0.6 / 0.7$ & $0.3 / 0.3$ & $0.2 / 0.0$ & $0.001 / 0.44$ \\
$S_{90}$ & $2.5 / 3.4$ & $0.2 / 0.3$ & $0.2 / 0.2$ & $0.001 / 0.007$ \\
$S_{180}$ & $3.2 / 4.1$ & $0.2 / 0.3$ & $0.2 / 0.2$ & $0.001 / 0.003$ \\
Compliance & & & & \\
$\left(10^{-4} \mathrm{~mm} / \mathrm{N}\right)$ & & & & \\
$C_{-180}$ & $52 / 28$ & $19 / 17$ & $9 / 3$ & $0.02 / 0.30$ \\
$C_{u 0}$ & $354 / 564$ & $71 / 93$ & $37 / 0$ & $0.01 / 0.60$ \\
$C_{10}$ & $308 / 441$ & $58 / 101$ & $20 / 9$ & $0.09 / 0.42$ \\
$\mathrm{C}_{180}$ & $62 / 66$ & $21 / 19$ & $0 / 0$ & $0.80 / 0.50$ \\
\hline
\end{tabular}

Subjects were measured six times each day on 10 or 11 subsequent days, respectively, by the same observer; $p$ values were obtained by testing the hypothesis that the theoretical day means were equal according to a one-way analysis of variance (random model).

which observer 1 was involved. Observer 1 generally showed the highest mean values for the normal knee. Observer 3 was the observer who provided the findings of Table 2 . Comparing the variation of the estimates of the different observer means with the pooled SD, we may conclude, with some caution, that the systematic differences between the theoretical observer means are not large relative to the SD of a single observer.

We questioned whether differences also existed between the SDs from different observers. With the exception of $S_{90}$ and $S_{180}$ for the unstable knee, this could not be established, using Bartletts' test for homogeneity of variances (generally, $p>0.05$ ).

Finally, Table 5 shows the results of comparing seven subjects before and after lumbar anesthesia. Shown are the means and SD of the average parameter values of both knees before and after anesthesia. Although the absolute means for most parameters were slightly larger with anesthesia than without (with the exception of $S_{90}$ and $C_{u 0}$ ), the differences were not significant according to Student's $t$ tests of paired observations ( $\mathrm{p}>0.05$ ).

\section{DISCUSSION}

In the accuracy tests using the RSP-measurement system, pure AP translations of tibia relative to femur did not occur in drawer tests, even if the thigh and the foot were fixed as well as possible. Femoral motions occurred within the soft tissues, even if the thigh holder was pressed hard on the upper leg. In addition, some ankle motion was possible in the foot holder. As a result, significant flexion-extension and tibial rotations occurred in

TABLE 4. Means, $S D$, and pooled $S D$ in five shift and compliance parameters

\begin{tabular}{|c|c|c|c|c|c|c|c|c|}
\hline \multirow[b]{2}{*}{ Parameter (unit) } & \multicolumn{5}{|c|}{ Observer: mean $(\mathrm{SD})(\mathrm{n}=5 \times 5)$} & \multirow{2}{*}{$\begin{array}{l}\text { Pooled } \\
\text { SD }\end{array}$} & \multirow{2}{*}{$\begin{array}{c}\mathrm{p} \text { Values } \\
\text { overall } \\
\text { hypothesis }\end{array}$} & \multirow{2}{*}{$\begin{array}{c}\text { (Nearly) } \\
\text { significant } \\
\text { pairs (p value) }\end{array}$} \\
\hline & 1 & 2 & 3 & 4 & 5 & & & \\
\hline \multicolumn{9}{|l|}{ Stable knee (R) } \\
\hline$S_{-180}(\mathrm{~mm})$ & $-6.9(0.7)$ & $-6.6(0.8)$ & $-5.7(0.6)$ & $-5.6(0.4)$ & $-5.7(0.1)$ & 0.6 & 0.003 & $\begin{array}{l}1-3(0.06) \\
1-4(0.03) \\
1-5(0.04)\end{array}$ \\
\hline$C_{-180}\left(10^{-4} \mathrm{~mm} / \mathrm{N}\right)$ & $110(23)$ & $105(36)$ & $89(16)$ & $81(30)$ & $78(17)$ & 24 & 0.21 & none \\
\hline$C_{u 0}\left(10^{-4} \mathrm{~mm} / \mathrm{N}\right)$ & $560(80)$ & $450(170)$ & $560(80)$ & $510(70)$ & $510(60)$ & 90 & 0.39 & none \\
\hline$C_{180}\left(10^{-4} \mathrm{~mm} / \mathrm{N}\right)$ & $112(28)$ & $96(24)^{a}$ & $91(19)$ & $58(18)^{a}$ & $67(16)^{b}$ & 22 & 0.02 & $1-4(0.04)$ \\
\hline$S_{180}(\mathrm{~mm})$ & $4.5(0.7)$ & $3.8(0.9)$ & $4.3(0.4)$ & $3.7(0.4)$ & $3.8(0.5)$ & 0.6 & 0.15 & none \\
\hline \multicolumn{9}{|l|}{ Unstable knee (L) } \\
\hline$S_{-180}(\mathrm{~mm})$ & $-8.6(1.2)$ & $-9.0(1.2)$ & $-8.5(0.3)$ & $-7.6(1.0)$ & $-8.5(0.5)$ & 0.9 & 0.27 & none \\
\hline$C_{-180}\left(10^{-4} \mathrm{~mm} / \mathrm{N}\right)$ & $130(25)$ & $118(30)$ & $105(15)$ & $109(23)$ & $99(26)$ & 24 & 0.31 & none \\
\hline$C_{u 0}\left(10^{-4} \mathrm{~mm} / \mathrm{N}\right)$ & $1080(50)$ & $960(200)$ & $1060(130)$ & $1070(140)$ & $1310(140)$ & 130 & 0.01 & $2-5(0.02)$ \\
\hline$C_{180}\left(10^{-4} \mathrm{~mm} / \mathrm{N}\right)$ & $114(24)$ & $92(17)^{a}$ & $104(16)^{a}$ & $119(23)^{a}$ & $96(19)^{c}$ & 20 & 0.33 & none \\
\hline$S_{180}(\mathrm{~mm})$ & $9.1(1.2)$ & $8.1(2.0)$ & $8.6(0.4)$ & $9.4(0.7)$ & $9.8(0.5)$ & 1.2 & 0.21 & none \\
\hline
\end{tabular}

${ }^{a}$ Only four values available; ${ }^{b}$ only two values available; ${ }^{c}$ only three values available.

Parameters were measured five times each by five different observers on one day in one patient (one normal, one ligament-deficient knee); $p$ values were obtained by testing the hypothesis of equal theoretical observer means according to a one-way analysis of variance (fixed model), and the significantly different observer pairs, together with the level of significance, according to the multiple comparison model of Scheffé. 
TABLE 5. Means and SD of average shift and compliance parameters of both knees in seven volunteers before and after lumbar anesthesia

\begin{tabular}{|c|c|c|c|c|c|}
\hline \multirow[b]{2}{*}{ Parameter } & \multicolumn{2}{|c|}{$\begin{array}{c}\text { Before } \\
\text { anesthesia } \\
(\mathbf{n}=7)\end{array}$} & \multicolumn{2}{|c|}{$\begin{array}{c}\text { After } \\
\text { anesthesia } \\
(\mathrm{n}=7)\end{array}$} & \multirow[b]{2}{*}{$p$ value } \\
\hline & Mean & SD & Mean & (SD) & \\
\hline \multicolumn{6}{|l|}{ Shift (mm) } \\
\hline$T_{90}$ & 5.2 & (2.3) & $5.3^{a}$ & $(2.3)$ & 0.68 \\
\hline $\mathrm{T}_{180}$ & 6.5 & $(3.0)$ & 6.6 & $(3.0)$ & 0.51 \\
\hline$S_{-180}^{100}$ & -1.7 & $(0.7)$ & -1.8 & $(0.8)$ & 0.85 \\
\hline$S_{-90}$ & -1.5 & $(0.7)$ & $-1.6^{a}$ & $(0.8)$ & 0.87 \\
\hline$H_{0}^{-00}$ & 0.6 & (1.1) & 0.8 & $(0.6)$ & 0.54 \\
\hline$S_{90}$ & 3.7 & (2.3) & 3.6 & (1.8) & 0.86 \\
\hline$S_{180}$ & 4.7 & (3.1) & 4.9 & $(2.8)$ & 0.73 \\
\hline \multicolumn{6}{|l|}{$\begin{array}{l}\text { Compliance } \\
\left(10^{-4} \mathrm{~mm} / \mathrm{N}\right)\end{array}$} \\
\hline$C_{-180}$ & $20^{b}$ & (26) & $38^{c}$ & (16) & 0.09 \\
\hline$C_{u 0}$ & 600 & (217) & 580 & (201) & 0.69 \\
\hline$C_{10}$ & 403 & (198) & 461 & (198) & 0.16 \\
\hline$C_{180}$ & $60^{c}$ & (24) & $111^{c}$ & $(80)$ & - \\
\hline
\end{tabular}

${ }^{a}$ Only six paired values available; ${ }^{b}$ only 5 paired values available; conly four paired values available.

$\mathrm{p}$ values were obtained by testing the hypothesis that anesthesia had no effect, according to paired Student's $t$ test.

the knee while the AP drawer test was executed. Usually, the AP drawer is rather loosely defined as a relative translation of the tibia in the AP direction. This definition is unique only when the tibia moves relative to the femur in a purely translational mode. Because rotations actually occur as well, the use of the accurate RSP measurement system requires a more precise definition. A choice was made, in this case, for the displacement between the tuberosity of the tibia and a point in the femur between the condyles, approximately where the helical axes of the knee flexion are located. When we determined motion in this way, and compared it with the values found with the laxity tester, we found errors of $\sim 10 \%$ in the total maximal shifts of the intact knee. These relative errors were smaller when the ligaments were cut. Although notable proximal-distal translations of the patella occurred, relative to the femur, their effects on the results were small. AP displacements of the patella relative to the femur were negligible.

Evidently, the laxity of the knee specimens was larger than is usually found in vivo (this was also concluded repeatedly by others, e.g., 12). Hence, the motion excursions obtained in the RSP measurements are not entirely realistic. Nevertheless, they illustrate two important points. First, it is evi- dent that an external thighholder is not effective in suppressing these motions completely; second, a differential measuring method can be effective in circumventing this problem. The important question is how these flexion and tibial rotation motions affect the validity of the test for particular diagnoses of ligament insufficiencies. It is a question that deserves further experimental and clinical research.

The reproducibility of the drawer test depends on a combination of errors, related either to the device itself, to the observer, or to the subject. Because of the segmental linearization of the curves obtained in the test subjects, the random errors are partially eliminated. Device-related random errors in the individual measurement points may occur because of the limitations in the resolution of the force and displacement registrations $(2.2 \mathrm{~N}$ and $0.35 \mathrm{~mm}$, respectively) and as a consequence of temperature fluctuations or drift in the electronic circuits. Once the subject is positioned in the apparatus, the results of the measurements can only be influenced by the force application rate, the time lapse between AP cycles (both related to viscoelastic effects), and by the muscle relaxation state of the subject. Although muscle relaxation is manually tested during the AP loading cycle and repeated tests (Fig. 2) show a high degree of reproducibility (provided that a full preconditioning cycle is made), imprecisions due to these effects cannot be completely discarded. Nevertheless, the most important observer-related errors are probably caused by differences between positioning procedures. This expectation is supported by the results of the positioning and measurement repeated five times on 12 normal subjects, which showed SD values of up to $0.9 \mathrm{~mm}$ for $S_{0}$, and only up to $0.3 \mathrm{~mm}$ for $H_{0}$. The former has no effect on the precision of the test, being a reference-determining parameter, but does give an indication of the reproducibility of the subject positioning procedure. The latter, the $H_{0}$, is a measure of viscoelastic effects in the knee. Its relatively low SD indicates that these effects play only a minor role in the overall errors.

Further support for the expectation that viscoelastic effects are negligible relative to the other errors is found in the results of the tests repeated 25 times on one subject by five different observers, in which no clear time effect was found. The importance of the positioning procedure was further em- 
phasized when the findings of different observers were compared (Table 5).

Although some significant between-day variations for the compliance parameters were found, most variations were negligible relative to the within-day variations (Table 3). A between-day variation in most of the shift parameters, however, could not be neglected. Because no clear time effect was found in the test involving different observers measuring one patient, it is unlikely that the between-day variation in shift parameters was observer related. Hence, it must have been either subject related or device related. Most likely, it was an effect of temperature differences or drift in the electronic parts of the displacement transducers (full-scale calibration). This would indeed affect the shifts more than the slopes.

There is no doubt that the results of the test can potentially be jeopardized by muscle activation of the subject; this can be clearly shown on the monitor. The expectation that by proper instruction and control this effect plays no important role in practice relative to positioning effects was supported by the comparison of tests before and after lumbar anesthesia (Table 5), which did not result in significant differences. A slightly higher laxity in the anesthetized subjects was found consistently, suggesting that significant differences could have been detected with more observations.

Overall, in the normal knee, using this particular laxity tester, a within-day variation on the order of $5 \%(\mathrm{CV})$ can be expected in the total laxity, between $-180 \mathrm{~N}$ and $+180 \mathrm{~N}$ and between $-90 \mathrm{~N}$ and $+90 \mathrm{~N}$. The anterior shifts for $90 \mathrm{~N}$ and $180 \mathrm{~N}$ were reproducible to $\sim 10 \%$; the posterior shifts were reproducible to $\sim 15 \%$. The within-day variation of the anterior and posterior compliances for $\pm 180 \mathrm{~N}$ was on the order of $30-40 \%$; the zeroforce compliances were $\sim 20 \%$. These relative errors were generally somewhat lower in unstable knees, because in that case the absolute parameter values were higher whereas the absolute errors were on the same order of magnitude.

Acknowledgment: This research project was sponsored in part by the Nederlandse Sport Totalisator.

\section{REFERENCES}

1. Blankevoort L, Huiskes R, Lange A de: Helical axes along the envelop of passive knee joint motion. Transactions of the 32nd Annual Orthopaedic Research Society. 1986

2. Bromage Ph.R. Epidural Analgesia. Philadelphia, W.B. Saunders, 1978

3. Butler DL, Noyes FR, Grood ES: Ligamentous restraints to anteriorposterior drawer in the human knee. $J$ Bone Joint Surg 62-A:259-270, 1980

4. Daniel DM, Malcom LL, Losse G, Stone ML, Sachs R, Burks R: Instrumented measurements of anterior laxity of the knee. I Bone Joint Surg 67-A:720-726, 1985

5. Fukubayashi T, Torzilli PA, Sherman MF, Warren PF: An in-vitro biomechanical evaluation of anterior-posterior motion of the knee. J Bone Joint Surg 64-A:258-264, 1982

6. GENUCOM: Documentation and personal communication. AAOS Commercial Exhibits, Las Vegas, 1985

7. Hsieh H-H, Walker PS: Stabilizing mechanisms of the loaded and unloaded knee joint. J Bone Joint Surg 58-A:8793, 1976

8. Huiskes $R$, Dijk $R$ van, Lange A de, Woltring HJ, Rens ThJG van: Kinematics of the human knee joint. In: Biomechanics of Normal and Pathological Human Articulating Joints, ed by N Berme, AE Engin, KM Correia da Silva, Dordrecht, Martinus Nijhoff, 1985, pp 165-187

9. Jacobson K: Gonylaxometry. Acta Orthop Scand 194: (suppl), 1981

10. Kennedy JC, Fowler PJ: Medial and anterior stability of the knee. J Bone Joint Surg 53-A:1257-1270, 1971

11. Lange A de, Kauer JMG, Huiskes R: Kinematic behavior of the human wrist joint: A roentgen-stereophotogrammetric analysis. J Orthop Res 3:56-64, 1985

12. Markolf KL, Graff-Radford A, Amstutz HC: In-vivo knee stability. A quantitative assessment using an instrumented clinical testing apparatus. J Bone Joint Surg 60-A:664-674, 1978

13. Markolf KL, Kochan A, Amstutz HC: Measurement of knee stiffness and laxity in patients with documented absence of the anterior cruciate ligament. $J$ Bone Joint Surg 66-A:242-253, 1984

14. Müller W: The Knee: Form, Function and Ligament Reconstruction. New York, Springer Verlag, 1983

15. Scheffé H: The Analysis of Variance. New York, Wiley, 1959

16. Selvik G: A Roentgenstereophotogrammetric Method for the Study of the Kinematics of the Skeletal System. Dissertation, AV-Centralen, Lund, Sweden, 1974

17. Shino K, Ohta, N, Horibe S, Ono K: In-vivo measurement of A-P instability in the ACL disrupted knees and in the postoperative knees. Transactions of the 30th Annual ORS:394, 1984

18. Theil $\mathrm{H}$ : A rank-invariant method of linear and polynomial regression analysis III. Proc $R$ Dutch Acad Sci A53:13971412,1950

19. Torzilli PA, Greenberg RL, Insall J: An in-vivo biomechanical evaluation of anterior-posterior motion of the knee. $J$ Bone Joint Surg 63-A:960-968, 1981

20. Winter DA: Biomechanics of Human Movement. New York, Wiley, 1979 